\title{
How human abilities affect component skills in word processing
}

\author{
MARC M. SEBRECHTS and JOSEPH G. DECK \\ Wesleyan University, Middletown, Connecticut \\ and \\ RICHARD K. WAGNER and JOHN B. BLACK \\ Yale University, New Haven, Connecticut
}

\begin{abstract}
Even simple tasks such as basic word processing can be quite complex. This paper argues that such tasks can be decomposed into a set of component skills and suggests how user abilities affect performance based on those skills. Special attention is given to spatial memory tests. It is argued that these tests actually assess two separable abilities: visual search and retention of order information. Skill acquisition can be facilitated by taking these abilities into account in system design: The importance of searching is decreased by providing context searches in which the system locates items in a text, and the importance of remembering the order of command sequences is improved by decreasing procedural complexity.
\end{abstract}

Despite claims of system simplicity, novice users still have substantial difficulties with rather basic word processing tasks (Lewis \& Mack, 1982). The degree of difficulty depends on individual user abilities, so performance varies widely across users for any given system. Egan, Bowers, and Gomez (1982), for example, demonstrated that errors in performance on a line-oriented editor were highly correlated with reading ability, spatial memory, and age. Further work with a full-screen editor (Gomez, Wheeler, Egan, Sharma, \& Gruchacz, 1983) again demonstrated strong effects of both reading ability and spatial memory.

In order to improve performance through enhanced training or system design, we need additional evidence concerning how user abilities are related to the component skills that word processing comprises. In particular, we are concerned with the role of spatial memory in using screen-based editors that attempt to decrease spatial requirements on users. The strategy is to train novices on a larger range of word processing tasks than is typically studied at any one time, including all of the tasks usually required for basic editing. We describe the component skills involved and attempt to relate individual abilities to these components.

\section{METHOD}

Materials

Two basic word processing systems were used: the IBM Displaywriter and the relevant portions of the UCSD Pascal

This research was supported by a grant from the IBM Corporation. However, the views expressed in this paper are those of the authors and are not necessarily endorsed by IBM. Send correspondence and reprint requests to: Marc M. Sebrechts, Department of Psychology, Wesleyan University, Middletown, CT 06457.
P-system running on a Terak 8510/A. Both systems are primarily menu driven, providing the user with a list of options from which a selection is made. The Displaywriter uses arbitrary code letters associated with each item in a menu; the UCSD system uses the first letter of key words (e.g., E for EDIT and F for FILE). (See Sebrechts, Deck, \& Black, 1983, for a detailed description.)

Pre- and Posttests

All subjects received a series of individual abilities tests either before or after training on word processing:

Typing test-Subjects were given a standard typing test both before and after training. The test consisted of a $2-\mathrm{min}$ warm-up and a 2-min typing test. Typing speed consisted of the number of five-letter words typed per minute, not counting words that contained errors.

Computer Operator Aptitude Battery-This test (Science Research Associates, 1973) includes three subparts: sequence recognition, which requires subjects to order items in terms of their order of occurrence; format checking, which requires subjects to perform string conversions based on a set of simple rules; and logical thinking, which asks subjects to fill in missing information in flowcharts.

Building Memory subtest-This is a test from the French kit (Ekstrom, French, \& Harman, 1976). Subjects study a map containing a series of buildings and then try to recall the locations of the buildings.

Picture-Number subtest-This is a paired-associate learning task that uses pictures and numbers. It is a general measure of associative learning from the French kit (Ekstrom et al., 1976).

Nelson-Denny Reading Test (1973)-Three subtests are taken from this test: reading speed, reading comprehension, and vocabulary. These are highly correlated with general reasoning ability.

\section{Procedure}

Subjects came into the laboratory on 5 consecutive days, for approximately $2 \mathrm{~h}$ each day. On the 1 st day, subjects were given the Computer Operator Aptitude Battery and the Building Memory test.

On the 2nd and 3rd days, subjects were trained on basic word processing tasks: creating, saving, revising, listing, and printing files. Twelve subjects used the IBM Displaywriter, and 
12 used the UCSD P-system. Half of the subjects on each system used a text manual; the others used a diagrammatic manual. Subjects were told to go through the materials sequentially. The experimenter was presented during the training, but did not give assistance unless the subjects were unable to continue on their own. Responses and times were recorded.

The 4 th and 5 th days included performance tests and three ability tests. The performance tests required subjects to create, revise, and restructure a memo, as well as to perform a series of editing tasks using commands specified by the experimenter. On the 4 th day, the subjects were not allowed to refer to the manuals. On the 5 th day, the subjects performed similar tasks and were allowed to use the manuals if they so desired. The ability tests were the Nelson-Denny test, a typing test, and the PictureNumber paired-associate test.

\section{Subjects}

Twenty-four subjects participated in the experiment. The subjects were students at Yale, Wesleyan, or Rutgers, a high school teacher, and a librarian. None of the subjects had had experience with word processing.

\section{RESULTS}

\section{Performance Measures}

All subjects completed all training exercises. Nevertheless, when they were required to use the skills learned, subjects showed substantial difficulty. On tasks that were like those the subjects had learned during training, the error rate was about $20 \%$. For tasks that required transfer of learning to new tasks, such as exchanging columns of text, error rates were as high as $50 \%$ of the tasks attempted.

When subjects were told the commands to use, error rates dropped substantially. However, a number of subjects continued to produce errors, particularly for complex commands such as REPLACE in Pascal, which requires the subjects to type $R$, then type the word to be replaced in delimiters, then type the new word within delimiters.

A detailed description of error types is given elsewhere (Galambos, Wikler, Black, \& Sebrechts, 1983). Our analysis of errors, however, can be described in terms of the following set of information processing components that the ability to use screen-oriented word processors comprises:

(1) Locate the modification to be made on paper.

(2) Store the modification in short-term memory.

(3) Find the appropriate region of text on the screen.

(4) Recall the modification command.

(5) Locate the cursor for the specific command.

(6) Issue the specific command.

(7) Verify the modification.

This basic description is based on our analysis, as well as on that of Egan and Gomez (1983). The components are similar to "operators" used by Card, Moran, and Newell (1980) to describe expert behavior. We have chosen this level of description because it captures the major types of errors that we have observed in our subjects. It can therefore be used as a framework for describing how user abilities might influence performance on component processes.

\section{Abilities}

The correlations between abilities and test performance are shown in Table 1 . The ability measures are the ones described in the Methods section. Test performance is a composite of the number of modifications completed in the two longer (and more reliable) tasks assigned to subjects during testing. Both of these tasks involve editing a multipage document. In one case, the subjects are simply told to perform the required modifications; in the other, they are told which commands to use. The data are standardized within each computer system (Displaywriter or UCSD P-system) and then combined.

As Table 1 shows, three abilities have substantial correlation with performance: Nelson-Denny, format checking, and sequence recognition. The first of these, Nelson-Denny, is a measure of reading ability. This variable accounts for about $25 \%$ of the variance in measured performance. This correlation is consistent with the results reported by Egan, Bowers, and Gomez (1982). To some extent, it probably reflects the general reading efficiency required for an editing task. In addition, measures of verbal ability tend to be correlated with other measures of general reasoning, and thus Nelson-Denny scores may indicate general facility with a number of the components we have mentioned. These same issues would come into play in different types of editors, so we would not expect that system design changes could eliminate this aspect of individual differences. Of course, to the extent that the system is made easy to use, the range of differences in performance would be expected to decrease.

Previous results have suggested an important role for spatial memory, but here the effect appears to be limited. In contrast to previous research, our study includes more complicated systems with a larger range of commands. This suggests that spatial memory may have an effect only with simple systems. In addition, whereas other work on screen editors uses special function keys, our system allows context searching, so that the system can assume some of the functions of searching. In terms of the components mentioned, this decreases the need for the third component-namely, finding the region of the page where the correction is to be made.

Another potentially important difference is the density of modifications in a task. The difficulty of a

Table 1

Correlation of the Ability Measures With Performance on the Word Processing Tests

$\begin{array}{lc}\text { Nelson-Denny Reading } & .52^{*} \\ \text { Building Memory } & .27 \\ \text { Picture-Number } & -.05 \\ \text { Sequence Recognition } & .42^{*} \\ \text { Format Checking } & .40^{*} \\ \text { Logical Thinking } & .29 \\ \text { Typing Speed } & .30\end{array}$

${ }^{*} p<.05$. 
search is a function of the density of items in a given location, and both the first component (locating the modification on the paper) and the third component (locating the modification region on the screen) require this type of search. The research that found an effect of spatial memory (Egan et al., 1982; Gomez et al., 1983) apparently utilized a relatively dense set of modifications (one modification every 1.5 lines of text; Egan, personal communication, February 21, 1983); in our study, the modifications required are relatively sparse (one modification every 4 lines). Thus, the role of spatial memory can in part reflect the difficulty of visual search on the typed page or on the computer screen.

Another possibility is that spatial memory reflects a more general ability to retain the order of items. In this case, the Building Memory test would actually be capturing some of the variance included in the format checking and sequence recognition tasks. This notion is supported by Egan and Gomez's (1983) finding of a relationship between Building Memory score and a subject's ability to formulate the proper command sequence (component 6 in our description). Further evidence is provided by our finding that Building Memory scores are correlated with performance on format checking $(r=.40)$.

Performance differences ascribed to spatial memory may, in fact, reflect the difficulty in retaining specific ordered sequences as specified by a set of rules. This is indicated by the correlations of format checking and sequence recognition with performance. Format checking accounts for about $11 \%$ of the variance independently of the effects of Nelson-Denny. However, sequence recognition adds only $2 \%$ to these two predictors. In sum, the two tests jointly indicate that sequence production based on rule sequences is an important ability for editing.

This suggests that users will have particular problems with procedures that require a sequence of commands, and that is precisely what we found. Although subjects seemed to perform quite well on the first three components, they frequently placed the cursor in the wrong position for a specific command or failed to generate the correct sequence (components 5 and 6).

\section{CONCLUSIONS}

The results suggest that sophisticated word processing systems are still difficult to learn for many users and that some of those difficulties may be inherent in the task. Difficulties with component skills based on reading ability or on task characteristics, such as error density, may persist in any system.

There are other areas, however, in which systems can be designed to accommodate user characteristics. Spatial memory skills actually seem to reflect two distinct components. On the one hand, these skills include basic processes of spatial segregation. The sensitivity of performance to these segregation abilities can be mini- mized in menu-driven, context-searching systems that can to some extent decrease the search requirements (particularly component 3 ). On the other hand, spatial memory also appears to reflect a basic ability to maintain the order of specific sequences. A full-screen editor, in many cases, can reduce such procedures. In a lineoriented editor, the command sequence itself must include information about the location of an item to be modified. Thus, in generating a command, the user must remember the appropriate sequence for specifying location as well as the modification to be made. In a screen editor, this complexity can be reduced because the user can locate where the change is to be made (component 5) and then generate the appropriate command sequence (component 6 ).

In the design of screen editors, it is important to take advantage of the spatial layout to minimize procedural complexity. Special attention should be given to designing simple command sequences to facilitate a user's generation of the appropriate command (component 6). However, caution should be exercised in making sure that a new procedural complexity is not inadvertently introduced into ways of positioning the cursor for different commands (component 5).

Finally, some of the skills we have described for word processing are probably of general importance for other computer skills. The format checking and sequence recognition tests are part of a computer operator aptitude test, and spatial abilities seem to be related to programming skills (Levy, Friestad, \& Vuchinich, 1982). An important topic for future research is to consider in more detail the nature of components in each of these computer skills.

\section{REFERENCES}

Card, S. K., Moran, T. P., \& Newell, A. (1980). Computer text-editing: An information-processing analysis of a routine cognitive skill. Cognitive Psychology, 12, 32-74.

Eqan, D. E., Bowers, C., \& Gomez, L. M. (1982). Learner characteristics that predict success in using a text editor tutorial. Proceedings of the Conference on Human Factors in Computer Systems (pp. 337-340). New York: Association for Computing Machinery.

Egan, D. E., \& Gomez, L. M. (1983). Components and correlates of computer text editing. Paper presented at the meeting of the American Educational Research Association, Montreal.

Exstrom, R., French, J,, \& Harman, H. (1976). Manual for kit of factor-referenced cognitive tests. Princeton, NJ: Educational Testing Service.

Galambos, J. A., Wikler, E. S., Black, J. B., \& Sebrechts, M. M. (1983). How you tell your computer what you mean: Ostension in interactive systems. In A. Janda (Ed.), Proceedings of the Conference on Human Factors in Computing Systems (pp. 182-185). New York: Association for Computing Machinery.

Gomez, L. M., Wheeler, E. A., Egan, D. E., Sharma, D. K., \& Gruchacz, A. M. (1983). Correlates of text editing using a screen editor: Spatial ability matters. Paper presented at the meeting of the American Educational Research Association, Montreal.

Levy, R. M., Friedstad, N., \& Vuchinich, R. (1982). Individual differences and adaptive behavior in reading letters from displays. Paper presented at the meeting of The Psychonomic Society, Minneapolis. 
LEwIs, C., \& Mack, R. (1982). Learning to use a text-processing system: Evidence from "think aloud" protocols. Proceedings of the Conference on Human Factors in Computer Systems (pp. 387-392). New York: Association for Computing Machinery. Nelson-Denny Reading Test. (1973). Boston, MA: Houghton Mifflin.
Science Research Associates. (1975). Computer operator aptitude battery. Chicago, IL: Science Research Associates.

Sebrechts, M. M., Deck, J. G., \& Black, J. B. (1983). A diagrammatic approach to computer instruction for the naive user. Behavior Research Methods \& Instrumentation, 15, 200-207. 